Check for updates

Cite this: RSC Adv., 2017, 7, 46969

Received 28th July 2017

Accepted 18th September 2017

DOI: $10.1039 / c 7 r a 08351 c$

rsc.li/rsc-advances

\section{Enhanced separation and antifouling performance of reverse osmosis membrane incorporated with carbon nanotubes functionalized by atom transfer radical polymerization $\dagger$}

\author{
Man Zhao, ${ }^{\text {ac }}$ Shancan Fu, ${ }^{\text {tb }}$ Huifeng Zhang, ${ }^{a}$ Hai Huang, ${ }^{a}$ Yangyang $\mathrm{Wei}^{\mathrm{a}}$ \\ and Yushan Zhang (iD *a
}

In this paper, an approach to obtain mixed-matrix reverse osmosis (RO) membranes with enhanced separation and antifouling performance is described. Atom transfer radical polymerization (ATRP) was first introduced for functionalization of multiwalled carbon nanotubes (MWCNTs) with polyacrylamide (PAAm) structure. Subsequently, MWCNTs were incorporated into polyamide (PA) thin film composite (TFC) membranes by interfacial polymerization. The dispersion of MWCNTs in aqueous solution and compatibility between MWCNTs and polymeric matrix were effectively improved with PAAm. Membrane surface morphology, hydrophilicity and charge properties were characterized by SEM, AFM, static water contact angles and zeta potential measurement. Moreover, the effect of above properties on separation and antifouling performance of membranes was investigated. Results showed that water flux of the optimal membrane incorporated with MWCNTs grafted at $6 \mathrm{~h}(\mathrm{MM}-6 \mathrm{~h})$ was $34 \%$ higher than that of virgin PA membrane. More importantly, $\mathrm{NaCl}$ rejection of $\mathrm{MM}-6 \mathrm{~h}$ membrane was $98.9 \%$, which is higher than that of virgin PA membrane and the membrane incorporated with oxidized MWCNTs. In addition, surface modified membranes showed excellent fouling resistance to BSA. The flux recovery of MM-6h membrane was about $97.6 \%$ after cleaning, while that of virgin PA membrane was only $79.2 \%$. This work proves that MWCNTs functionalized by ATRP have potential application in simultaneously improving separation and antifouling performance of TFC RO membranes.

\section{Introduction}

Aromatic polyamide (PA) thin film composite (TFC) membranes prepared by interfacial polymerization have been widely used in alleviating shortage of water resources by converting brackish water and seawater into clean water. ${ }^{\mathbf{1 , 2}}$ Unfortunately, the development of PA TFC membranes has encountered a bottleneck because of the trade-off limit between its permeability and selectivity. ${ }^{3}$ In addition, fouling is another remarkable obstacle. It can lead to a sharp time-dependent decline in permeate flux, resulting in reduction of membrane life and increase in

${ }^{a}$ Institute of Seawater Desalination and Multipurpose Utilization, State Oceanic Administration, Tianjin 300192, China. E-mail: manzhaocrystal@hotmail.com; Tel: +86-02287898130

${ }^{b}$ College of Engineering and Technology, Tianjin Agriculture University, Tianjin 300384, China

${ }^{c}$ College of Chemistry and Chemical Engineering, Ocean University of China, Qingdao 266100, Shandong, China

$\dagger$ Electronic supplementary information (ESI) available: HAADF-STEM-EDS elemental mapping images (Fig. S1). The dispersion photographs of MWCNTs in pure water at $25{ }^{\circ} \mathrm{C}$ (Fig. S2), static water contact angles of RO membranes (Fig. S3), results of separation performances of RO membranes (Table S1) are in the ESI. See DOI: 10.1039/c7ra08351c operation and maintenance costs. ${ }^{4}$ Therefore, there is an urgent requirement for the development of excellent separation RO membranes with durability against foulants.

Some strategies have been proposed to improve the performance of PA TFC membranes, e.g., optimization of interfacial polymerization conditions, ${ }^{5}$ tailoring of synthetic monomer, ${ }^{6,7}$ addition of functional additive ${ }^{8,9}$ and surface modification of RO membranes. ${ }^{\mathbf{1 0 - 1 5}}$ Although the above approaches can regulate performance of RO membranes by physical and chemical management, they usually focus on the improvement of a single property while the other properties of membranes are improved ineffectively or even weakened in some cases. For example, membrane coating a hydrophilic material on the surface leads to significantly improved antifouling behavior and similar or improved rejection, but is always accompanied by loss of water flux, which results from the penetration of coating solution into open pore structures. ${ }^{16}$ Therefore, it is still a challenge to develop RO membranes with improved water flux, salt rejection and fouling resistant.

Recently, the application of nanomaterials in preparation of mixed-matrix PA TFC membranes has provided a new perspective to improve performance of RO membranes. ${ }^{17}$ Some 
studies have demonstrated that PA TFC membranes incorporated with nanoparticles, e.g., $\mathrm{SiO}_{2},{ }^{18,19}$ silver nanoparticles, ${ }^{20,21}$ $\mathrm{TiO}_{2},{ }^{22}$ zeolite $^{23}$ and carbon nanotubes (CNTs), ${ }^{24-28}$ show increased water flux, salt rejection and/or excellent antifouling property due to improvement in surface properties of PA separation layer. Among these nanomaterials, CNTs have attracted much attention in fabricating filtration membranes because of their intrinsic 1-dimensional pore structure for superior transport capability. ${ }^{29}$ Unfortunately, CNTs always present an agglomerate state due to their strong $\pi-\pi$ interaction, ${ }^{30}$ leading to nonselective defects or large gaps that reduce membrane selectivity. ${ }^{31}$ Moreover, compatibility of CNTs with polymeric matrix is another problem that determines membrane performance. ${ }^{32}$ Therefore, it is necessary to apply surface functionalization to improve dispersion and compatibility of CNTs in polymeric matrix.

Extensive ultrasonic treatment in a mixture of concentrated nitric and sulfuric acid is always used to prevent aggregation of CNTs. Meanwhile, the introduction of carboxylic groups allows CNTs to disperse well in aqueous solution, ${ }^{33}$ which contributes to stable CNTs within polymeric matrix. ${ }^{34}$ Although membranes with carboxylic CNTs show enhanced water permeability, good antifouling and anti-oxidative properties, weak noncovalent interactions between CNTs and polymeric matrix also lead to a decreased salt rejection, which is still a great obstacle in the application of CNTs to membrane separation. New methods are demanded to improve both dispersion of CNTs and its interactions with polymeric matrices. Atomic transfer radical polymerization (ATRP) has the advantage of a highly controllable polymerization process, applicability to a wide range of vinyl monomers and mild polymerization conditions ${ }^{35}$ which is an effective strategy of CNT functionalization to obtain amount of functional groups, e.g., amide, hydroxyl and epoxy groups. ${ }^{30}$ Polyacrylamide (PAAm) exhibits the properties of hydrophilicity and functionality, thus allowing for excellent dispersion and stronger interactions with PA matrices.

In this work, we employed ATRP to modify MWCNTs by grafting hydrophilic and functional PAAm. Mixed-matrix RO membranes were fabricated by interfacial polymerization. The surface morphology, hydrophilicity and charge properties as well as separation and antifouling performance of membranes incorporated with functionalized MWCNTs were investigated and compared with those containing oxidized MWCNTs and those without any MWCNTs.

\section{Experimental}

\subsection{Materials and reagents}

Multiwalled carbon nanotubes (MWCNTs, purity $\geq 95 \%$ ) were purchased from Deco Island Gold Technology Co., Ltd, Beijing, China, and the average diameter and average length of a MWCNT was $20-30 \mathrm{~nm}$ and $10-30 \mu \mathrm{m}$, respectively. Ethylene glycol (EG, Tianjin Jiangtian Chemicals, 98\%) was purified by distillation under vacuum. Acrylamide (AAm, Tianjin Jiangtian Chemicals, 98\%) was recrystallized from acetone before use. Copper(I) bromide (CuBr, Alfa Aesar, 99\%) was purified by stirring it with acetic acid for $12 \mathrm{~h}$, and then was washed with ethanol and diethyl ether. Finally, it was dried under vacuum at $75{ }^{\circ} \mathrm{C}$ for 3 days. $n$-Hexane (Tianjin Jiangtian Chemicals, 98\%) as an organic solvent was first dried with calcium hydride overnight and then filtered with filter paper. Sulfuric acid $\left(\mathrm{H}_{2} \mathrm{SO}_{4}, 98 \%\right.$, Tianjin Jiangtian Chemicals), nitric acid $\left(\mathrm{HNO}_{3}\right.$, $60 \%$, Tianjin Jiangtian Chemicals), hydrogen peroxide $\left(\mathrm{H}_{2} \mathrm{O}_{2}\right.$, $30 \%$, Tianjin Jiangtian Chemicals), thionyl chloride $\left(\mathrm{SOCl}_{2}\right.$, 98\%, Tianjin Jiangtian Chemicals), trimethylamine (TEA, 99\%, Tianjin Jiangtian Chemicals), sodium chloride ( $\mathrm{NaCl}, 99 \%$, Tianjin Jiangtian Chemicals), 2-bromoisobutyryl bromide (BIBB, Heowns, 98\%), $N, N, N^{\prime}, N^{\prime \prime}, N^{\prime \prime}$-pentamethyldiethylenetriamine (PMDETA, Heowns, 99\%), 4-dimethylaminopyridine (DMAP, Heowns, 98\%), anhydrous copper(II) bromide $\left(\mathrm{CuBr}_{2}\right.$, Heowns, 98\%), trimesoyl chloride (TMC, Heowns, 99\%), 1,3phenylenediamine (MPD, Aladdin Agent, 99\%), and camphorsulfonic acid (CSA, Aladdin Agent, 99\%) were used as received. Polysulfone supporting membrane (PS, molecular weight cut-off $32000 \mathrm{Da}$ ) was supplied by the Pureach Technology Co., Ltd, Beijing, China. The thickness of PS support layer was $48 \mu \mathrm{m}$ and pure water flux was $230 \mathrm{~L} \mathrm{~m}^{-2} \mathrm{~h}^{-1}$ at the applied pressure of 1 bar and temperature of $25{ }^{\circ} \mathrm{C}$. Bovine serum albumin (BSA, Beijing Solarbio Science \& Technology Co., Ltd.) was stored at $-20^{\circ} \mathrm{C}$ prior to use. Deionized (DI) water was obtained from a water purification system (Synergy, Millipore, USA).

\subsection{MWCNT functionalization and characterization}

Fig. 1(a) illustrates functionalization procedure of MWCNTs. In order to introduce ATRP initiator onto the surface of MWCNTs, $1.0 \mathrm{~g}$ MWCNTs were first treated with mixture acid $\left(\mathrm{H}_{2} \mathrm{SO}_{4} /\right.$ $\mathrm{HNO}_{3}=3 / 1, \mathrm{v} / \mathrm{v}$ ) at $45{ }^{\circ} \mathrm{C}$ for $1 \mathrm{~h}$ and refluxed at $65^{\circ} \mathrm{C}$ for $3 \mathrm{~h}$, and then MWCNTs were treated with a mixture of $\mathrm{H}_{2} \mathrm{SO}_{4}$ and $\mathrm{H}_{2} \mathrm{O}_{2}(4 / 1, \mathrm{v} / \mathrm{v})$ at $70{ }^{\circ} \mathrm{C}$ for $2 \mathrm{~h}$. The MWCNTs were washed thoroughly to a neutral $\mathrm{pH}$ with DI water at every step, and then were dried in a freeze dryer. The obtained carboxylic MWCNTs (MWCNT-COOH) were ultrasonicated for $30 \mathrm{~min}$ in $50 \mathrm{~mL}$ $\mathrm{SOCl}_{2}$, and then were refluxed with pyridine as acid binding agents at $70{ }^{\circ} \mathrm{C}$ for $24 \mathrm{~h} .30 \mathrm{~mL}$ EG was added after removing $\mathrm{SOCl}_{2}$, and then the mixture was refluxed at $120^{\circ} \mathrm{C}$ for $48 \mathrm{~h}$ in anaerobic atmosphere. The hydroxyl modified MWCNTs (MWCNT-OH) were obtained finally. $0.45 \mathrm{~g}$ MWCNT-OH were ultrasonicated in $10 \mathrm{~mL} \mathrm{CH}_{2} \mathrm{Cl}_{2}$ with $0.1 \mathrm{~g}$ DMAP and $1 \mathrm{~mL}$ TEA. When the solution was cooled down to $0{ }^{\circ} \mathrm{C}, 1.5 \mathrm{~g}$ BIBB was added dropwise. Then, the reaction solution was stirred for 24 h. Finally, brominated MWCNTs (MWCNT-Br) were obtained.

PAAm was grafted onto MWCNT surface according to the following procedure: MWCNT-Br with surface-bound ATRP initiator ( $50 \mathrm{mg}), \mathrm{AAm}(0.36 \mathrm{~g}, 5 \mathrm{mmol}), \mathrm{CuBr}_{2}(0.33 \mathrm{mg}, 0.0025$ $\mathrm{mmol})$ and $\mathrm{H}_{2} \mathrm{O}(10 \mathrm{~mL})$ were added into a one-neck roundbottom flask $(25 \mathrm{~mL})$ successively. The reaction mixture was purged with argon for $15 \mathrm{~min}$ and then PMDETA $(8.68 \mathrm{mg}, 0.05$ mmol) was added. After another 15 min of argon bubbling, $\mathrm{CuBr}(4.50 \mathrm{mg}, 0.025 \mathrm{mmol})$ was added into the reaction mixture. The flask was then sealed, immersed into a water bath at $25{ }^{\circ} \mathrm{C}$, and stirred for different times, i.e., $3 \mathrm{~h}, 6 \mathrm{~h}$ and $12 \mathrm{~h}$. 
(a)

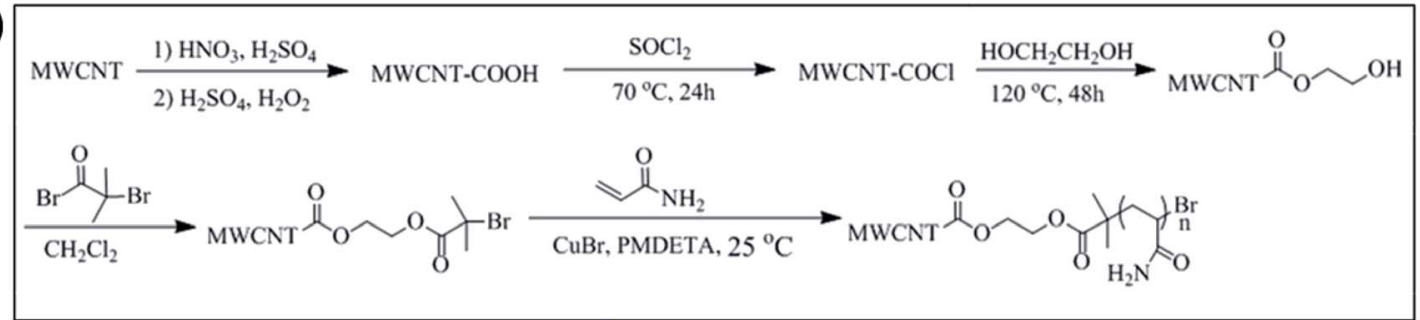

(b)

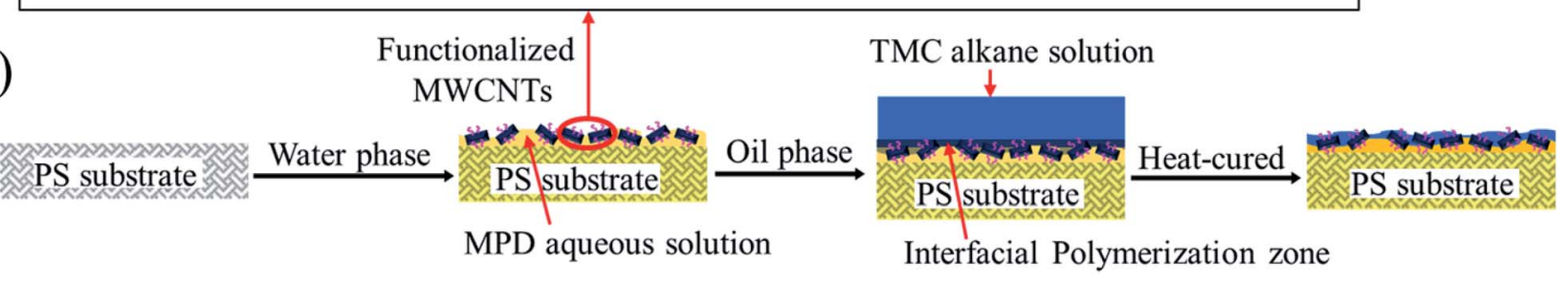

Fig. 1 (a) Chemical scheme for functionalization of MWCNTs and (b) schematic of interfacial polymerization process with functionalized MWCNTs.

The resulting solid product was washed with $\mathrm{H}_{2} \mathrm{O}$ and methanol, and then was dried at $40{ }^{\circ} \mathrm{C}$ under vacuum overnight to a constant weight. Based on grafting time, the functionalized MWCNTs were defined as PAAm- $3 \mathrm{~h}$, PAAm- $6 \mathrm{~h}$ and PAAm- $12 \mathrm{~h}$.

The morphologies of MWCNTs were characterized by scanning electron microscopy (SEM, Nova NanoSEM 450, Japan) and transmission electron microscopy (TEM, Hitachi H7650, Japan). The high-angle annular dark-field scanning TEM-energydispersive X-ray spectroscopy (HAADF-STEM-EDS) was performed on a Hitachi $\mathrm{H} 7650$ microscope operated at $200 \mathrm{kV}$. The chemical structure was characterized by Fourier transform infrared radiation (FTIR, Bio-rad FTS6000, USA) and thermal gravimetric analysis (TGA, Netzsch TG209, Germany). The size of MWCNT nanoclusters in aqueous solution was determined by dynamic light scattering (DLS, Zetasizer Nano ZSP, Malvern, UK).

\subsection{Preparation of $\mathrm{RO}$ membrane incorporated with MWCNTs}

Schematic of interfacial polymerization with functionalized MWCNTs is shown in Fig. 1(b). Interfacial polymerization on surface of a pre-cast PS substrate was conducted to form the PA selective layer. PS substrates were immersed into MPD aqueous solutions $(2 \mathrm{wt} \%)$ for $60 \mathrm{~s}$. A series of aqueous solutions were prepared with different amounts of functionalized MWCNTs grafted at $6 \mathrm{~h}$. Another series of aqueous solutions were prepared with functionalized MWCNTs grafted at various times $(3 \mathrm{~h}, 6 \mathrm{~h}, 12 \mathrm{~h})$. Then, the residual aqueous solution on substrate surface was drained off. Subsequently, alkane ( $n$-hexane) solution containing $0.1 \mathrm{wt} \%$ TMC was added to the MPD-rich substrate and then was drained off after $40 \mathrm{~s}$. The nascent membranes were dried at ambient conditions for $1 \mathrm{~min}$, and then heat-cured at $80{ }^{\circ} \mathrm{C}$ for $5 \mathrm{~min}$. The resulting membranes were stored in $\mathrm{NaHSO}_{3}$ solution (1 wt\%) to prohibit microbial growth. Based on grafting time of functionalized MWCNTs, the prepared mixed-matrix RO membranes were labeled as MM- $3 \mathrm{~h}$, MM-6h and MM-12h. Control experiments for virgin PA membrane and membrane incorporated with MWCNT-COOH were prepared and defined as PA and MM- $\mathrm{COOH}$, respectively.

The interaction between functionalized MWCNTs and TMC monomer was investigated by the following: $20 \mathrm{mg}$ MWCNTs grafted at $6 \mathrm{~h}$ were dispersed into organic phase ( $n$-hexane with $0.1 \mathrm{wt} \% \mathrm{TMC}$ ) by ultrasonic treatment for $20 \mathrm{~min}$. The resulting MWCNTs were rinsed with hexane several times and dried completely. X-ray photoelectron spectroscopy (XPS, K-alpha, Thermo Fisher, USA) was employed to analyze the interaction between MWCNTs and TMC.

\subsection{Membrane characterization}

The membrane samples were rinsed with DI water and dried under vacuum at $25{ }^{\circ} \mathrm{C}$ for $24 \mathrm{~h}$ prior to all characterizations. The surface morphologies were observed by SEM (Nova NanoSEM 450). Surface roughness of RO membranes was measured by a multimode atomic force microscopy (AFM, Nanoscope V MultiMode controller, Bruker, Germany) in air and quantitative analysis was in terms of measured root mean square (RMS) roughness from height profile of three-dimensional AFM images. Surface hydrophilicity of RO membranes was evaluated by static water contact angle using the sessile drop method with a goniometer (OCA20, Data Physics, Germany) at $25{ }^{\circ} \mathrm{C}$. Membrane samples were attached onto flat glass slides and $2 \mu \mathrm{L}$ of DI water were formed at the end of an "I"-shaped needle. Then, the flat glass slide was carefully elevated toward the droplet to deposit the droplet on the membrane surface. For each sample, at least three measurements were taken at different locations to obtain the average contact angle value. Surface zeta potential of RO membranes was measured by a SurPASS solid surface zeta potential analyzer (Anton Paar, Austria). Streaming potential measurements were carried out with a background electrolyte solution containing $1 \mathrm{mM} \mathrm{KCl}$ at $\mathrm{pH} 7.0$ and $25{ }^{\circ} \mathrm{C}$. The zeta potential of RO membranes was computed from the Helmholtz-Smoluchowski equation. 


\subsection{Separation performance tests}

Separation performance tests of RO membranes were conducted by a customized cross-flow RO cell. An aqueous solution containing $2000 \mathrm{ppm} \mathrm{NaCl}$ was used as the feed water at $25^{\circ} \mathrm{C}$. The membranes were initially equilibrated with DI water at 1.55 MPa for $1 \mathrm{~h}$ and then the volume of the permeate water was collected to calculate water flux $\left(J, \mathrm{~L} \mathrm{~m}^{-2} \mathrm{~h}^{-1}\right)$ by eqn (1). $\mathrm{NaCl}$ rejection $(R, \%)$ was evaluated by quantifying conductivity of the permeate water and feed water using a conductivity meter with eqn (2).

$$
\begin{gathered}
J=\frac{V}{S t} \\
R=\frac{C_{\mathrm{f}}-C_{\mathrm{P}}}{C_{\mathrm{f}}} \times 100
\end{gathered}
$$

where $t$ is the test time $(\mathrm{h}), V$ is the volume $\left(\mathrm{m}^{3}\right)$ of permeate water collected during the test, $S$ is the effective membrane area $\left(\mathrm{m}^{2}\right), C_{\mathrm{f}}$ and $C_{\mathrm{P}}$ are the salt concentrations in feed and permeate solution, respectively.

\subsection{Antifouling experiments}

Membrane antifouling performance was estimated via crossflow filtration of an aqueous solution containing $2000 \mathrm{ppm}$ $\mathrm{NaCl}$ and $200 \mathrm{ppm}$ foulant (BSA) at $25{ }^{\circ} \mathrm{C}$ and $1.55 \mathrm{MPa}$. The antifouling property was evaluated by monitoring permeation flux as a function of time. The membranes were first stabilized with $2000 \mathrm{ppm} \mathrm{NaCl}$ aqueous solution for $1 \mathrm{~h}$ and the average water flux was recorded as $J_{0}$. After that, a mixture with desired concentration of BSA was added to the feed tank. The permeate flux was monitored continuously during the testing period to investigate fouling behavior of RO membranes. After fouling for $12 \mathrm{~h}$, the membranes were rinsed with DI water for $1 \mathrm{~h}$, and the RO membranes were filtrated with $2000 \mathrm{ppm} \mathrm{NaCl}$ solution to determine recovery of water flux.

\section{Results and discussion}

\subsection{Chemical structure and morphology of functionalized MWCNTs}

Generally, high performance of mixed-matrix RO membranes requires homogeneous distribution of MWCNTs in polymeric matrix and excellent compatibility between MWCNTs and PA. However, CNTs are not easily dispersed well in the PA layer, which may affect membrane performance due to the high surface energy of raw MWCNTs. To solve this problem, ATRP is used to functionalize MWCNTs by grafting functional polymer, i.e., polyacrylamide (PAAm), as shown in Fig. 1.

Fig. 2 represents FTIR spectra of raw MWCNTs and functionalized MWCNTs. For raw MWCNTs, there are weak peaks at $2916 \mathrm{~cm}^{-1}$ and $2846 \mathrm{~cm}^{-1}$ corresponding to $\mathrm{sp}^{2} \mathrm{C}-\mathrm{H}$ and $\mathrm{sp}^{3}$ $\mathrm{C}-\mathrm{H}$ stretching bands, respectively, which originate from defects at both sidewalls and open ends of MWCNTs. The defects provide abundant reaction sites for modification to MWCNTs. The successful chemical modification of MWCNTs by acid is confirmed by characteristic peaks of $\mathrm{C}=\mathrm{O}$ at
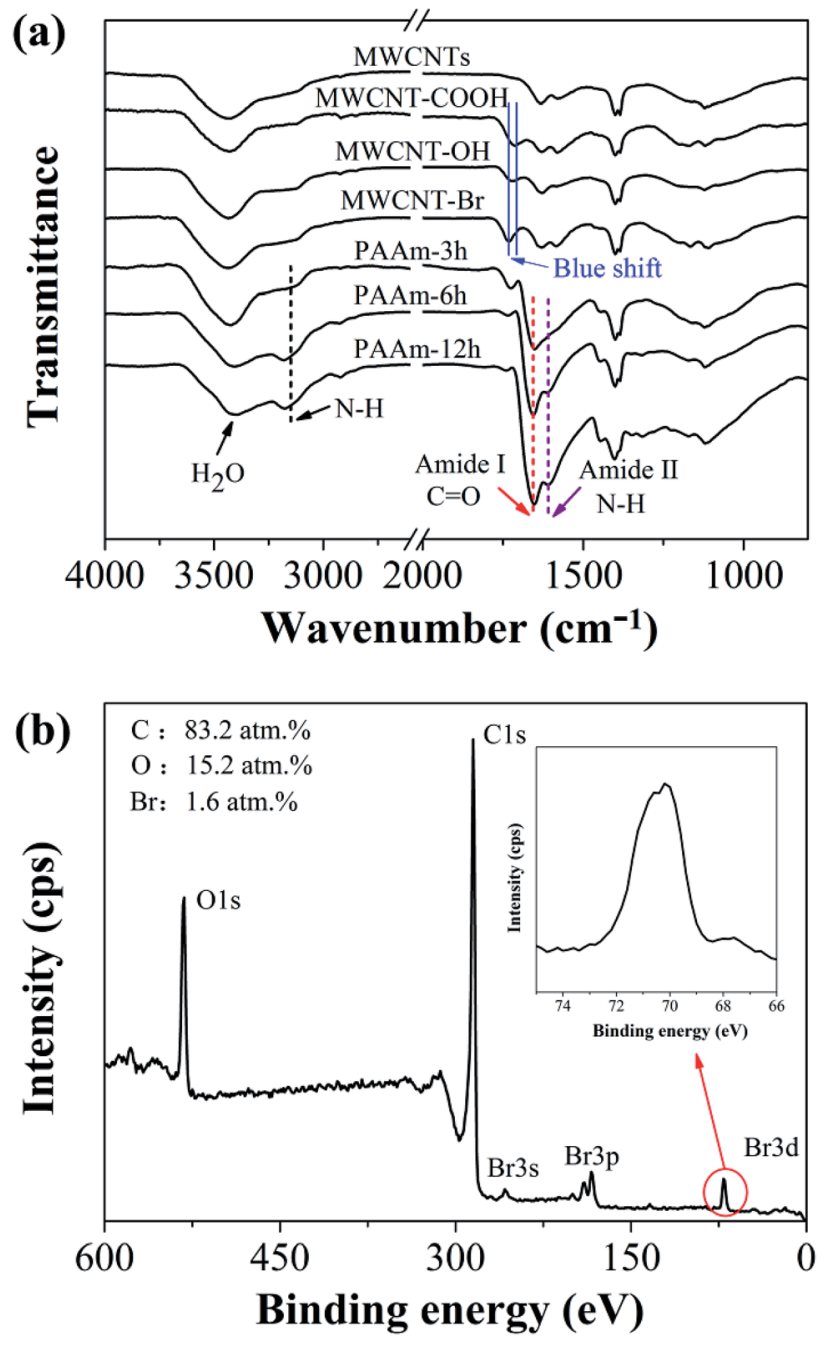

Fig. 2 (a) FTIR spectra of raw MWCNTs and functionalized MWCNTs; (b) XPS survey spectrum of MWCNT-Br, inset is enlarged image of $\mathrm{Br} 3 \mathrm{~d}$.

$1720 \mathrm{~cm}^{-1}$ and $\mathrm{C}-\mathrm{O}$ at $1060 \mathrm{~cm}^{-1}$ in the spectrum for MWCNTCOOH. ${ }^{36}$ The blue-shift of $\mathrm{C}=\mathrm{O}$ characteristic peak in FTIR spectra of MWCNT-OH and MWCNT-Br is ascribed to change in chemical environment around $\mathrm{C}=\mathrm{O}$, which further verifies the formation of ester carbonyl and hydroxyl groups anchored onto the surface of MWCNTs. The $\mathrm{C}-\mathrm{Br}$ characteristic peak is not observed in FT-IR spectra because of the weak vibrations, while the bromine peak ( $\mathrm{Br} 3 \mathrm{~d}$ peak at $70.7 \mathrm{eV}$ (ref. 37)) is observed in the XPS spectra and the atomic ratio of $\mathrm{Br} 3 \mathrm{~d}$ is $1.6 \%$, which demonstrates the introduction of ATRP initiator on MWCNT surface. The presence of two significant peaks, amide I band (around $1653 \mathrm{~cm}^{-1}, \mathrm{C}=\mathrm{O}$ stretching) and amide II bond (around $1604 \mathrm{~cm}^{-1}, \mathrm{~N}-\mathrm{H}$ stretching), in FTIR spectra of MWCNTs grafted with PAAm supports the existence of PAAm in all MWCNTs grafted at different times. Meanwhile, the peak around $3174 \mathrm{~cm}^{-1}$ in the spectra can be ascribed to asymmetric $\mathrm{N}-\mathrm{H}$ stretching bonds. ${ }^{38}$ Although one of the $\mathrm{N}-\mathrm{H}$ stretching bonds from the primary amide partially overlaps with the feature peak of $\mathrm{H}_{2} \mathrm{O}$, the existing characteristic peaks still verify the successful grafting of PAAm onto MWCNTs. In addition, the 
intensity of feature peaks from PAAm become stronger with increase in grafting time after infrared spectrum normalization.

In order to further ascertain successful functionalization of MWCNTs, thermal degradation behavior of MWCNTs was estimated by TGA. Fig. 3 displays the TGA curves of MWCNT samples under nitrogen atmosphere at a heating rate of $10{ }^{\circ} \mathrm{C} \mathrm{min}^{-1}$. It is observed that raw MWCNTs undergo practically negligible mass loss before $400{ }^{\circ} \mathrm{C}$. However, there is approximately $10 \%$ mass loss of MWCNT-COOH caused by decomposition of carboxylic group, which indicates the successful oxidation of MWCNTs. The mass loss increases to $15 \%$ after modification of EG, and there is about $19 \%$ mass loss of brominated MWCNTs, which is ascribed to the successful anchor of hydroxyl group and ATRP initiator sites onto MWCNTs. The thermal stability of modified MWCNTs is lower than that of raw MWCNTs, which is due to oxidation and modification on the surface of MWCNTs. In addition, TGA is used to estimate the quality of PAAm grafted onto MWCNT surface under different grafting times by weight loss values. At $600{ }^{\circ} \mathrm{C}$, it can be calculated that the weight gains of MWCNTs grafted at $3 \mathrm{~h}, 6 \mathrm{~h}$ and $12 \mathrm{~h}$ are 5\%,15\% and 24\%, respectively. Moreover, the above results correlate with the FTIR analysis and prove that functionalization degree depends greatly on grafting time.

Fig. 4 shows SEM and TEM images of MWCNTs before and after functionalization. Raw MWCNTs are highly entangled with each other as a result of van der Waals forces of attraction, ${ }^{39}$ whereas short and individual oxidized MWCNTs can be observed because of ultrasonic and acidification treatment. More importantly, compared with pristine ones, the sidewalls of oxidized MWCNTs become rougher and open ends appear. In addition, an amorphous layer coating on the surface of PAAm6h MWCNTs can be investigated clearly using high-resolution TEM. The thickness of the layer is about $5 \mathrm{~nm}$, which reveals the successful grafting of PAAm onto MWCNT surface by ATRP. ${ }^{40}$ More definite composition information of PAAm layer is provided by HAADF-STEM-EDS merged elemental mapping

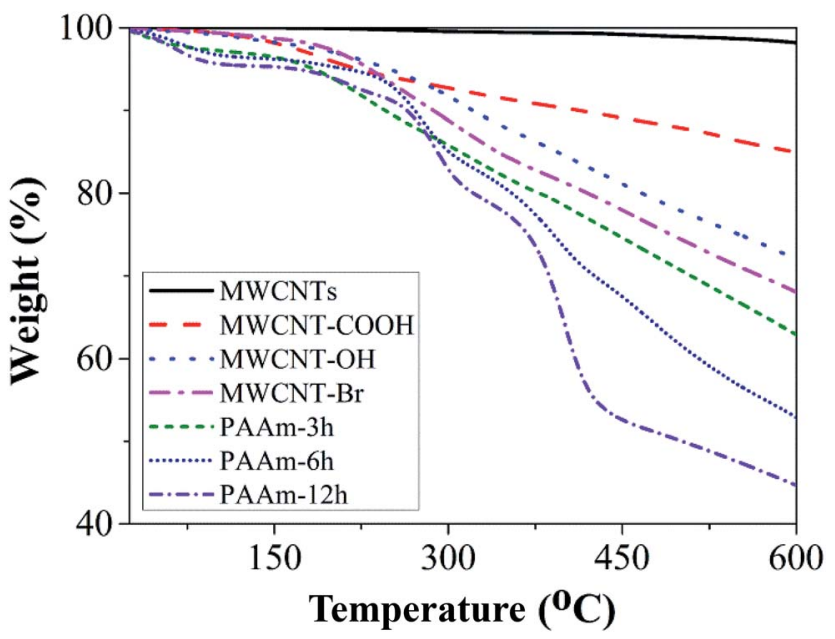

Fig. 3 TGA results of raw MWCNTs, modified MWCNTs and MWCNTs grafted with PAAm
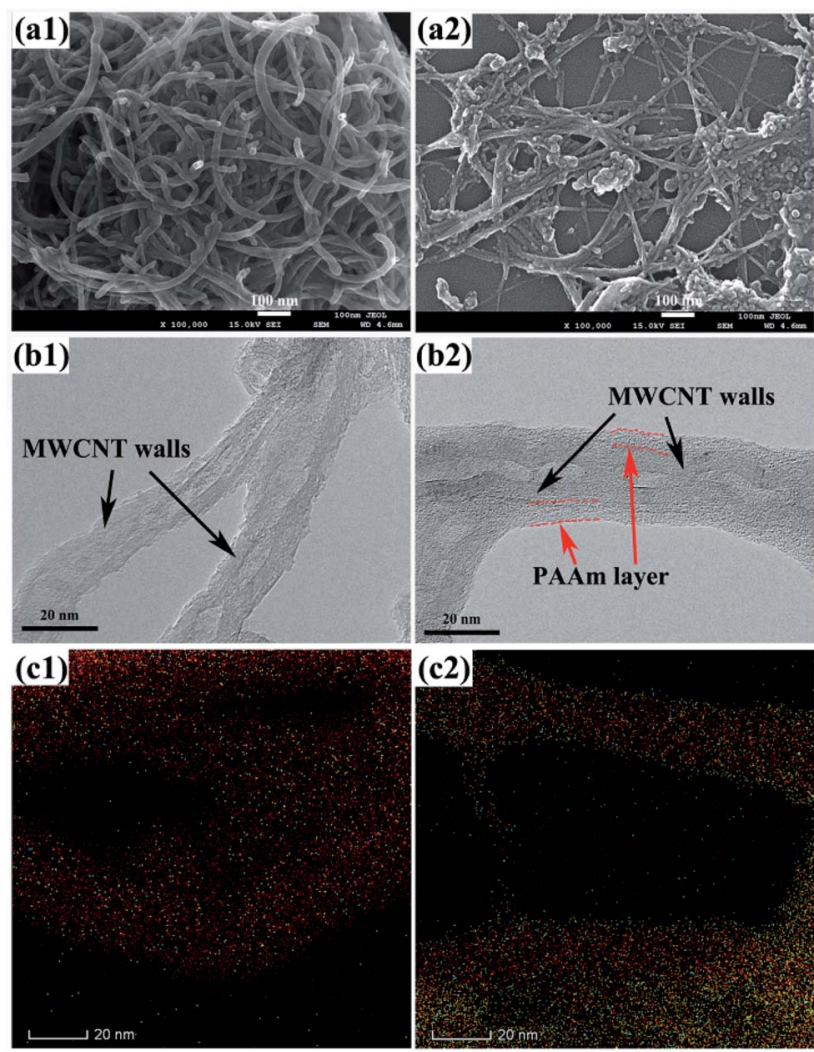

(c2)

Fig. 4 SEM images of (a1) raw MWCNTs and (a2) MWCNT-COOH; TEM images of (b1) MWCNT-COOH and (b2) PAAm-6h; HAADFSTEM-EDS merged elemental mapping images of (c1) MWCNT- $\mathrm{COOH}$ and (c2) PAAm-6h. (C-red, N-blue and O-yellow)

images (Fig. 4(c)). The detailed images are shown in Fig. S1. $\dagger$ It can be seen that the intensity of $\mathrm{N}$ and $\mathrm{O}$ in PAAm- $6 \mathrm{~h}$ is much higher than that in MWCNT-COOH, indicating that PAAm nanolayer exists on the surface of PAAm-6h.

\subsection{Physical properties of RO membrane surface}

Mixed-matrix RO membranes were prepared by typical interfacial polymerization, except that functionalized MWCNTs were included in the MPD aqueous solution. For investigating the influence of MWCNTs on the prepared membranes, physical properties of membrane surface, i.e., morphology, hydrophilicity and zeta potential, were characterized.

Surface morphologies of RO membranes were investigated by SEM, and the corresponding images are shown in Fig. 5. The PA membrane exhibits similar hill-and-valley microstructure as the morphology reported before. ${ }^{28}$ This hill-and-valley structure increases surface roughness, and the contact area between water and PA layer also increases, which is responsible for the excellent permeation property of the TFC membrane. After incorporation of MWCNTs, the surface morphology of mixedmatrix membranes undergoes changes compared to that of virgin PA membrane. This is probably because of the decreased diffusion rate of MPD for the incorporation of MWCNTs and the restricted motion behavior of MWCNTs with PAAm macromolecule chain. Moreover, dispersion ability of MWCNTs in 

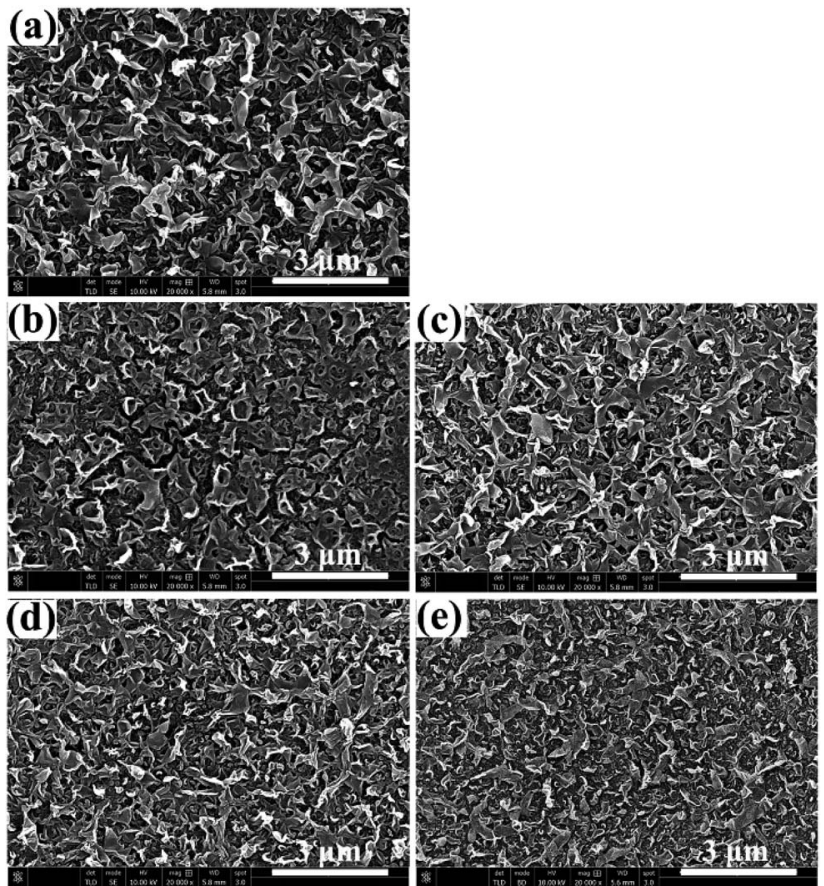

Fig. 5 SEM images of membrane surfaces of (a) PA, (b) MM-3h, (c) $M M-6 h$, (d) MM-12h and (e) MM-COOH

aqueous solution impacts surface morphology of mixed-matrix membranes. The aggregation phenomenon in aqueous solutions occur when MWCNTs are grafted for $3 \mathrm{~h}$ (Fig. S2†). As a result, surface morphology of MM-3h membrane changes greatly, as shown in Fig. 5(b).

AFM was also employed to investigate surface morphology of RO membranes. Fig. 6 shows the roughness of PA, MM-3h, MM6h, MM-12h and MM-COOH membrane. The virgin PA membrane exhibits relatively rough surface morphology with RMS of $82.3 \pm 2.1 \mathrm{~nm}$. After incorporating MWCNTs, the mixed-matrix RO membranes exhibit relatively smooth and flat surface. Notably, the RMS of MM-6h membrane is just $54.1 \pm 0.9 \mathrm{~nm}$. The smoother membrane surface is attributed to reduction in interfacial polymerization rate resulting from confined diffusion of MPD into oil phase. ${ }^{41}$ These results indicate that introduction of MWCNTs can increase the smoothness of the membrane surface, which is beneficial for antifouling property of RO membranes. ${ }^{35}$

The static water contact angle was employed to evaluate hydrophilic property of membrane surface. As shown in Table 1 and Fig. S3, $\uparrow$ contact angles of mixed-matrix membranes decrease with introduction of MWCNTs. Most functionalized MWCNTs show relatively high water dispersion stability (Table 2 and Fig. S2†), which indicates improved hydrophilicity of MWCNTs. Consequently, membrane surface hydrophilicity is enhanced due to incorporation of hydrophilic MWCNTs into the PA layer. ${ }^{26,42}$ Futhermore, the descendant of interfacial polymerization rate caused by decreased diffusion of MPD leads to more residual acyl chloride groups on the membrane surface, which will finally hydrolyze into carboxyl groups. ${ }^{41}$ Moreover, the influence of chemical composition on membrane surfaces (a1)

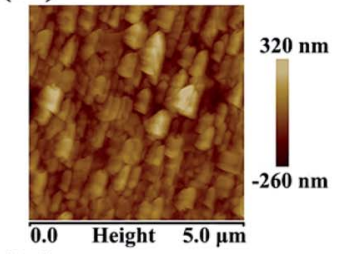

(b1)
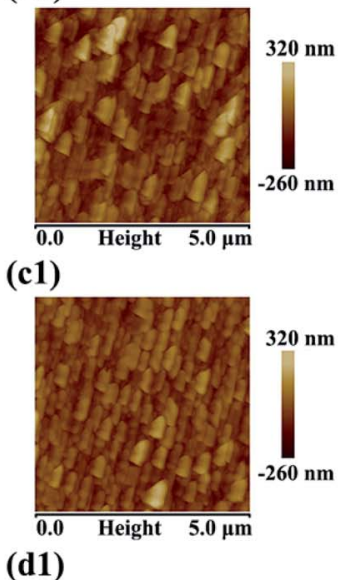

(d1)

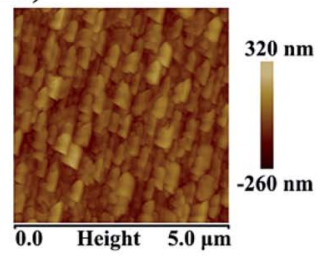

(e1)
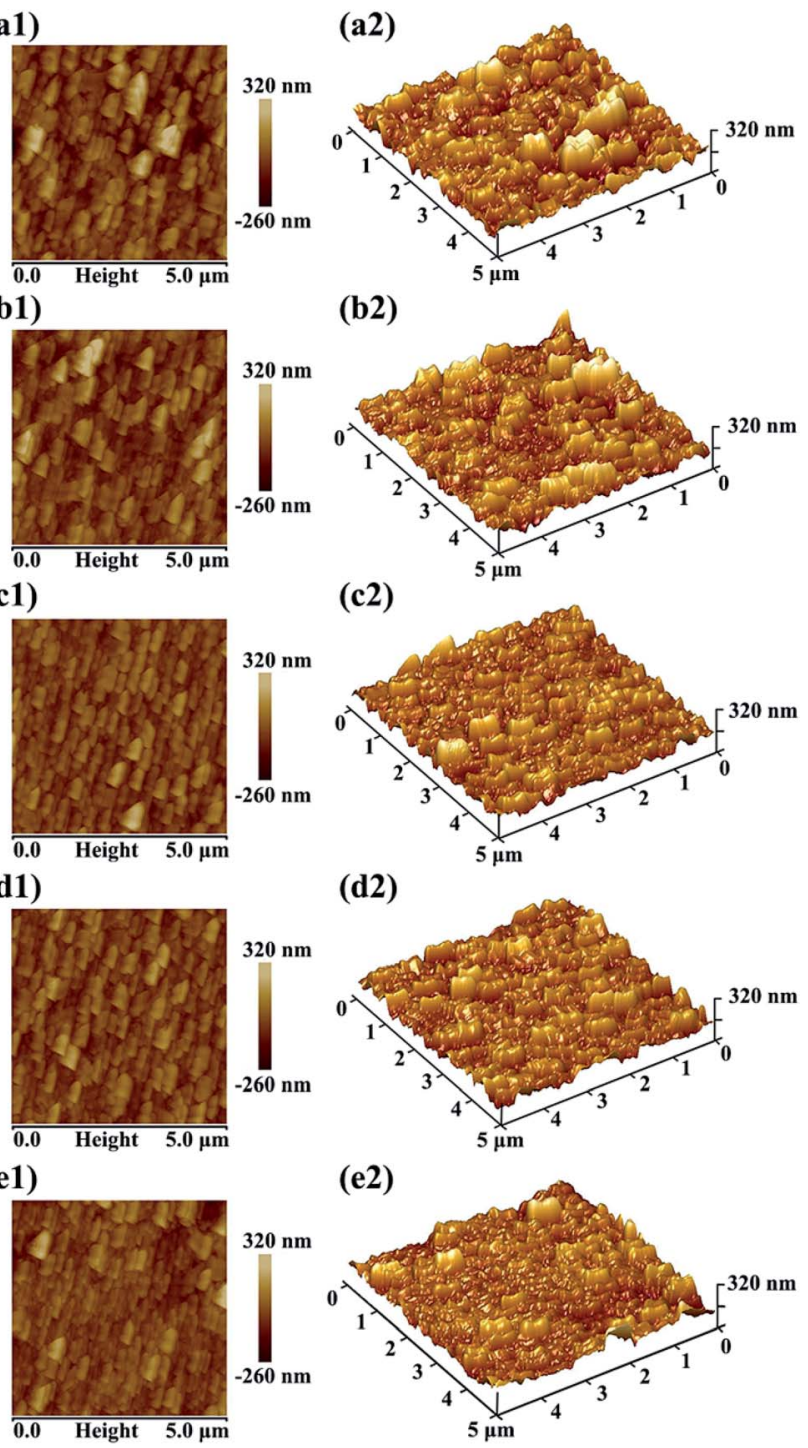

(d2)

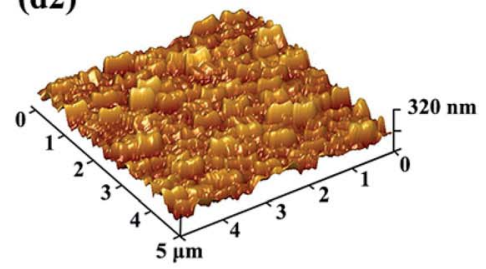

(e2)

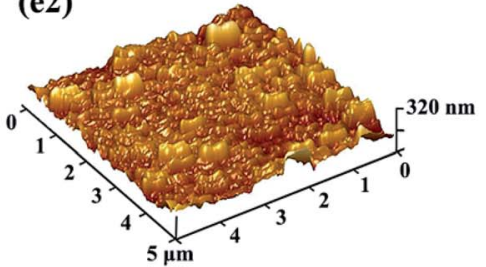

Fig. 6 AFM images of (a) PA, (b) MM-3h, (c) MM-6h, (d) MM-12h and (e) MM-COOH membranes.

may be greater than that of surface roughness. As a result, the contact angle decreases in spite of decrement in surface roughness. This similar phenomenon is also presented in previous reports. ${ }^{11,43}$ It is generally accepted that lower values of static water contact angles are indicative of higher hydrophilicity, which is beneficial to the formation of water layer on

Table 1 Summary of membrane surface roughness parameter, static contact angle and charge property data

\begin{tabular}{|c|c|c|c|}
\hline Membrane & RMS (nm) & $\begin{array}{l}\text { Static contact } \\
\text { angle }\left(^{\circ}\right)\end{array}$ & Zeta potential $(\mathrm{mV})$ \\
\hline PA & $82.3 \pm 2.1$ & $64.8 \pm 3.2$ & $-32.9 \pm 1.5$ \\
\hline MM-3h & $70.7 \pm 1.5$ & $57.8 \pm 1.1$ & $-38.3 \pm 2.0$ \\
\hline MM-6h & $54.1 \pm 0.9$ & $52.5 \pm 0.7$ & $-44.4 \pm 1.2$ \\
\hline MM-12h & $57.3 \pm 1.1$ & $48.0 \pm 2.8$ & $-41.4 \pm 1.8$ \\
\hline $\mathrm{MM}-\mathrm{COOH}$ & $56.4 \pm 0.5$ & $49.0 \pm 3.5$ & $-39.7 \pm 1.0$ \\
\hline
\end{tabular}


Table 2 Apparent size distribution of MWCNTs in aqueous solution

\begin{tabular}{ll}
\hline CNTs & $\begin{array}{l}Z \text {-Average } \\
(\mathrm{nm})\end{array}$ \\
\hline MWCNTs & 590.7 \\
PAAm-3h & 404.0 \\
PAAm-6h & 262.2 \\
PAAm-12h & 297.7 \\
MWCNT-COOH & 175.5
\end{tabular}

the membrane surface. This water layer provides a barrier to the membrane surface and effectively inhibits attachment of foulants onto the membrane surface. ${ }^{44}$ Therefore, the introduction of MWCNTs may favor the antifouling property of RO membranes.

Zeta potential of the prepared membranes was also investigated to characterize the effect of introducing MWCNTs on the surface charge of membranes, as listed in Table 1 . The virgin PA membrane has negative charge with a zeta potential of $-32.9 \pm$ $1.5 \mathrm{mV}$, which is due to the presence of carboxyl groups originating from partial hydrolysis of the acyl chloride unit of TMC during membrane preparation. After introducing MWCNTs, the mixed-matrix membranes exhibit increased negative charge density. The reason may be attributed to negative charge of functionalized MWCNTs at $\mathrm{pH}$ 7.0. Additionally, the zeta potential values of MM-6h membrane is slightly lower than that of MM-COOH membrane, which is mainly caused by the larger partial negative charge around oxygen atoms of PAAm between oxygen and nitrogen atoms of the amide groups, while the negative charge is equally shared between two oxygen atoms of the carboxyl groups. The negatively charged surface of mixedmatrix RO membranes will be responsible for resistance to foulants.

\subsection{Separation performance of mixed-matrix RO membranes}

Water permeability and selectivity of RO membranes were investigated with a cross-flow cell, and all the membranes were tested at least three times within the margins of error. It has been known that the amount of CNTs is a vital factor to separation performance of mixed-matrix membranes. ${ }^{26,27}$ Table $\mathrm{S} 1 \dagger$ lists water flux and salt rejection values of RO membranes prepared with various concentrations of PAAm-6h MWCNTs. When membrane was prepared with an aqueous solution containing 0.0025 wt\% PAAm-6h, water flux and $\mathrm{NaCl}$ rejection values were $38.4 \mathrm{~L} \mathrm{~m}^{-2} \mathrm{~h}^{-1}$ and $98.2 \%$, respectively. It is clear that the very small amount of CNTs did not obviously affect membrane performance. In contrast, when membranes were prepared with a larger amount of PAAM-6h, i.e., $0.0075 \mathrm{wt} \%$ and $0.01 \mathrm{wt} \%$, the water flux increased largely, while the salt rejection decreased significantly. For example, very large water flux of $105.7 \mathrm{~L} \mathrm{~m}^{-2} \mathrm{~h}^{-1}$ and very small $\mathrm{NaCl}$ rejection of $15.8 \%$ were observed from membrane prepared with aqueous solution containing $0.01 \mathrm{wt} \%$ PAAm-6h. This possibly results because excessive MWCNT aggregations, i.e., bundles, occur as a result of interactions among CNTs. ${ }^{25,31}$ Membrane prepared with

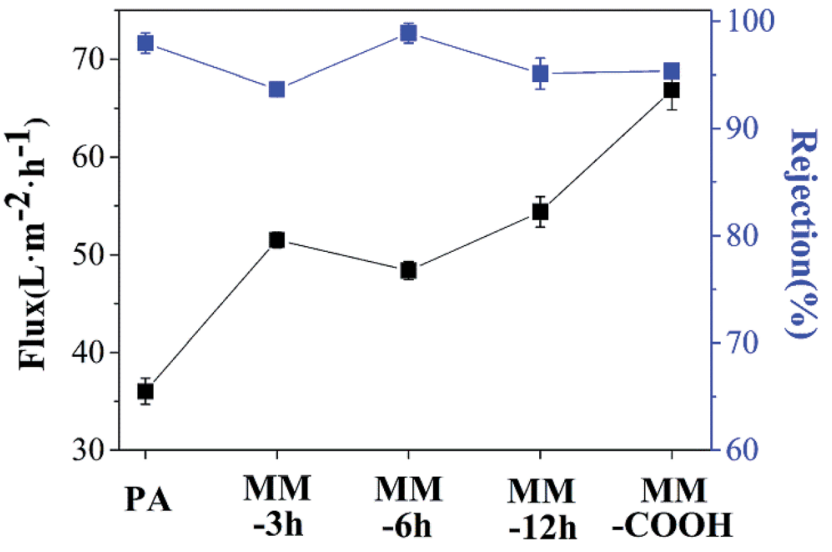

Fig. 7 Separation performance of RO membranes.

aqueous solution containing 0.005 wt $\%$ PAAm-6h exhibited an excellent separation performance and a detailed study will be conducted in the following sections. Therefore, $0.005 \mathrm{wt} \%$ was selected as the optimum concentration to prepare mixed-matrix membranes with other functionalized MWCNTs. The separation performance of these membranes is presented in Fig. 7.

Compared with the PA membrane without MWCNTs, water flux of mixed-matrix membranes increases from 36.0 to 51.5, 48.4, 54.4 and $66.9 \mathrm{~L} \mathrm{~m}^{-2} \mathrm{~h}^{-1}$. It has well been demonstrated that MWCNTs can act as extraordinary mass transport channels in polymeric matrix, ${ }^{45}$ which contributes to shortening of the path of water molecules, resulting in enhancement in permeation performance for mixed-matrix RO membranes. As discussed above, the introduction of MWCNTs can increase hydrophilicity of RO membranes and the hydrophilic nature facilitates rapid transport of water molecules, which plays an important role in water permeability enhancement. ${ }^{45}$ Moreover, the relatively smooth surface is responsible for improvement in water flux. ${ }^{46}$ Nevertheless, the membrane incorporated with PAAm functionalized MWCNTs shows a much lower water flux than the MM-COOH membrane (Fig. 7), which might be mainly due to the existing lower content of CNTs. After ATRP grafting, PAAm on MWCNTs make the CNT particles heavier than carboxylic MWCNTs. Therefore, the number of CNT particles inserted in PA matrix decreases due to application of constant mass fraction of MWCNTs in aqueous solution, leading to reduction in water transport channels after the formation of the PA layer.

After introducing MWCNTs, NaCl rejection of MM-3h and MM-12h was $93.7 \%$ and $95.1 \%$, respectively, which is lower than that of virgin PA membrane (97.9\%). For the MM-3h membrane, limited hydrophilic property of MWCNTs caused by the short grafting time was responsible for the aggregation of MWCNTs in aqueous solution (Fig. $\mathrm{S} 2 \dagger$ ), which results in lower selectivity performance compared with that of other membranes. For MWCNTs grafted for $12 \mathrm{~h}$, slight agglomeration of MWCNTs in aqueous solution appeared because of the long grafting time (Fig. S2 $\uparrow$ ), which may have a negative impact on salt rejection. Therefore, it can be concluded that appropriate grafting time is 
critical to selectivity performance of mixed-matrix RO membranes.

In particular, MM-6h membrane exhibits higher $\mathrm{NaCl}$ rejection in comparison with $\mathrm{MM}-\mathrm{COOH}$ membrane $(98.9 \%$ vs. $95.3 \%$ ). This result can be attributed to the excellent dispersion of MWCNTs in aqueous solution and compatibility of MWCNTs with polymeric matrix. After grafting PAAm using ATRP, the amide groups on MWCNT surface are not only responsible for improving dispersion of MWCNTs in aqueous solution but also for enhancing compatibility between MWCNTs and PA matrix via covalent bonding, as shown in Fig. 8. The apparent particle size distributions of raw MWCNTs and MWCNTs grafted PAAm at $6 \mathrm{~h}$ are $590.7 \mathrm{~nm}$ and $262.2 \mathrm{~nm}$, respectively, which verify the well dispersed state of PAAm-6h in aqueous solution. Given that amidation will change chemical state of $\mathrm{N}$-element from $-\mathrm{CONH}_{2}$ to $-(\mathrm{CO})_{2} \mathrm{HN}-$, a quantitative analysis is performed to estimate reaction extent by resolving characteristic $\mathrm{N} 1 \mathrm{~s}$ peak in the XPS spectra, and the result is shown in Fig. 9. The peak at $399.6 \mathrm{eV}$ is assigned to typical amide group $(\mathrm{N}-\mathrm{C}=\mathrm{O})$ from PAAm. ${ }^{47}$ The $\mathrm{O}=\mathrm{C}-\mathrm{N}-\mathrm{C}=\mathrm{O}$ group has a higher binding energy at $400.1 \mathrm{eV}$ due to the higher oxidation state of nitrogen, which is consistent with the binding energy of nitrogen in succinimide. ${ }^{48}$ Then the ratio of $-\mathrm{CONH}_{2}$ to $-(\mathrm{CO})_{2} \mathrm{HN}$ - can be calculated by integration of corresponding peak areas, which eventually equals to about $1.88: 1$. That is to say, $35 \%$ of amines from PAAm is consumed to form new amide bonds with TMC monomers during interfacial polymerization. Therefore, MWCNTs after functionalization are promising for chemically coupling with PA matrix via stable amide bonds, which is significant in improving the compatibility and thus the crosslinking degree of PA matrix. As a result, the interface defects channels between MWCNTs and PA matrix are partially eliminated. In addition, the MM-6h membrane exhibits a lower water flux value than the other MWCNT incorporated membranes, which may be caused by a relatively higher crosslinking degree between MWCNTs grafted at $6 \mathrm{~h}$ and PA. In general, the carboxyl group in MWCNT-COOH can also covalently bond to chloride groups in $\mathrm{TMC}^{43}$ which results in improved compatibility of

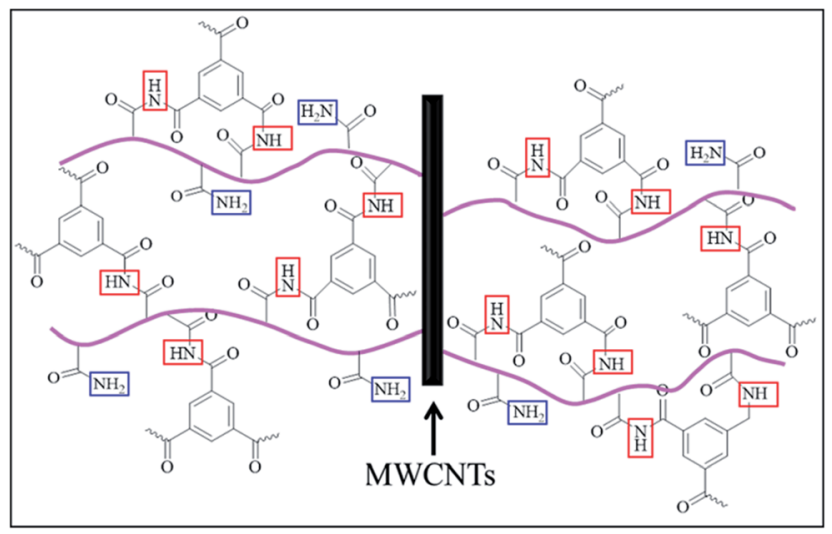

Fig. 8 Schematic of interaction between functionalized MWCNTs and TMC. The functional groups in boxes are the reacted products resulting from PAAm with TMC (red) and residual amine groups of PAAm (blue).

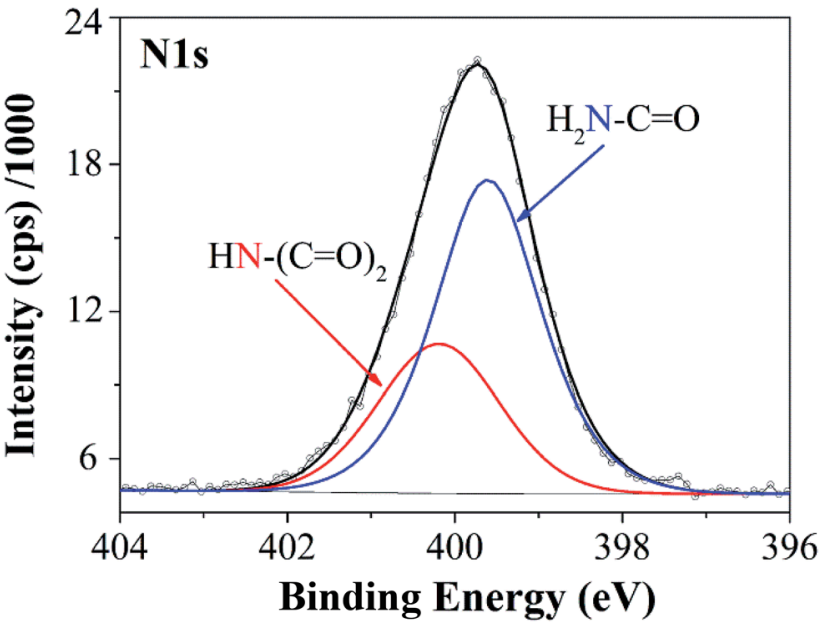

Fig. 9 XPS survey spectra of element $\mathrm{N}$ from MWCNTs of PAAm- $6 \mathrm{~h}$ after interaction with TMC.

carboxyl group in MWCNT-COOH with PA matrix. However, the number of functional groups $\left(\mathrm{NH}_{2}\right)$ in PAAm-6h is much larger than that of carboxyl groups in MWCNT-COOH, which can be verified by HAADF-STEM-EDS element mapping images in Fig. 4 and $\mathrm{S} 1 . \dagger$ As a result, the number of covalent bonds of PAAm- $6 \mathrm{~h}$ between MWCNTs and TMC are more than that of MWCNT$\mathrm{COOH}$. The number of covalent bonds are attributed to crosslinking degree of PA layer, which is beneficial for improving selectivity of RO membranes. ${ }^{49}$ Therefore, although both PAAm$6 \mathrm{~h}$ and MWCNT-COOH have the well dispersion state in aqueous solution (Fig. S2 $\dagger$ ), the salt rejection of MM-6h membrane is higher than that of $\mathrm{MM}-\mathrm{COOH}$ membrane. Table 3 compares the results of this study with that of previous papers.

\subsection{Antifouling property of mixed-matrix RO membranes}

Fouling resistance behavior of RO membranes was characterized by means of cross-flow tests with BSA as representative organic foulant. In order to evaluate the change in water flux with respect to operation time, normalized flux $J / J_{0}$ is plotted in Fig. 10. It can be seen that the mixed-matrix RO membrane has better antifouling property than the virgin PA membrane. When operation time increases to $12 \mathrm{~h}$, the water flux of virgin PA membrane decreases to $74.6 \%$, while that of MM-3h, MM- $6 \mathrm{~h}$ and $\mathrm{MM}-12 \mathrm{~h}$ membranes decreases to $80.7 \%, 91.4 \%$ and $89.1 \%$, respectively. More importantly, MM-6h membrane exhibits a similar antifouling behavior as MWCNT-COOH membrane, which indicates that a small amount of BSA is deposited on the membrane surface. As presented in Fig. 11, a compact BSA layer covering the whole surface of virgin PA membrane, while the MM- $6 \mathrm{~h}$ membrane exhibits a relatively clean surface and is partially covered with loosely deposited BSA layer.

The flux recovery was also investigated by hydraulic cleaning of membranes with DI water and the results are listed in Table 4. In general, mixed-matrix membranes exhibit much higher flux recovery than virgin PA membrane. In the best case, that of 
Table 3 Comparison of separation performance of mixed-matrix RO membranes in literature

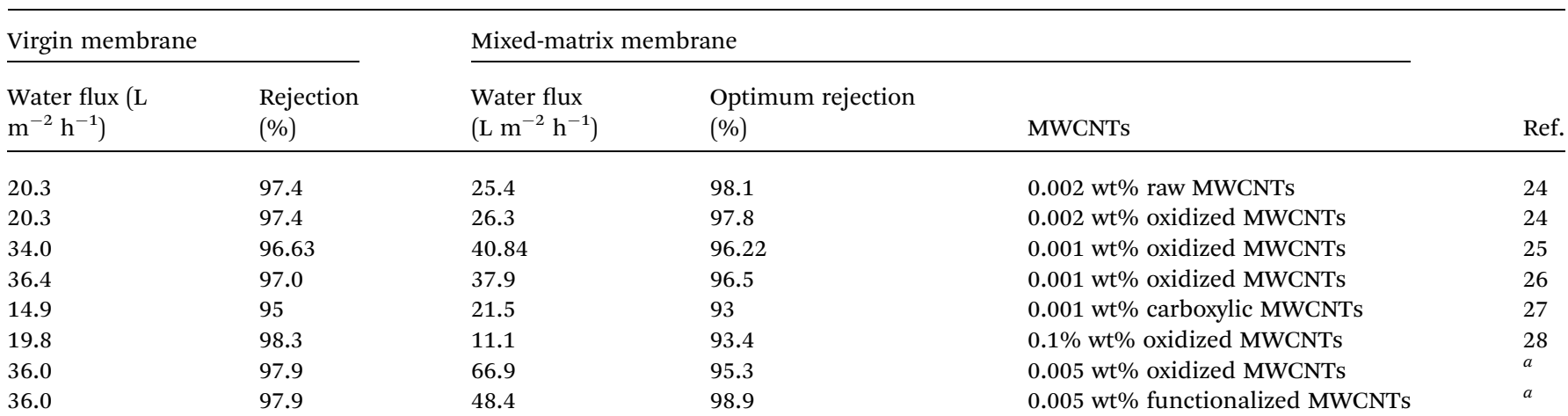

${ }^{a}$ Refers to this work.

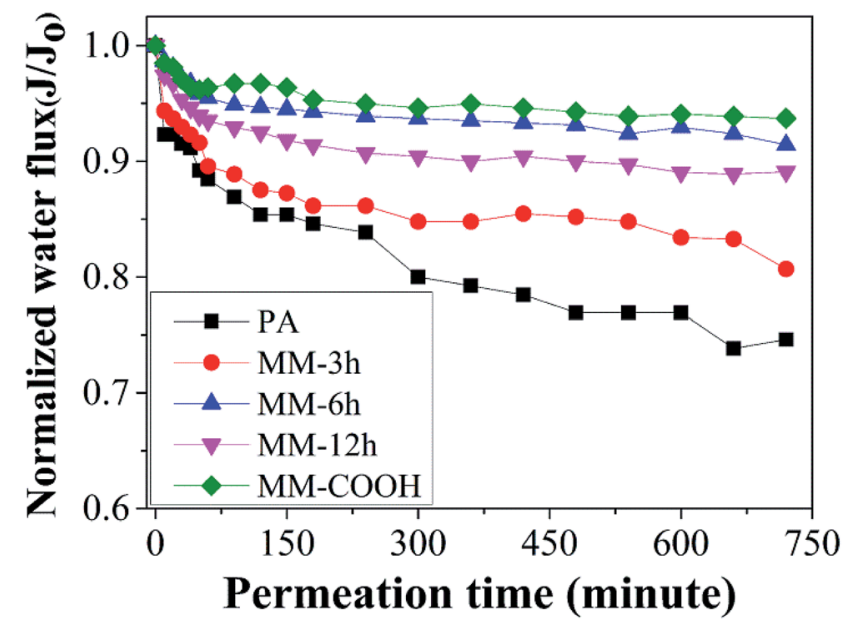

Fig. 10 Effects of BSA fouling on water flux of RO membranes.
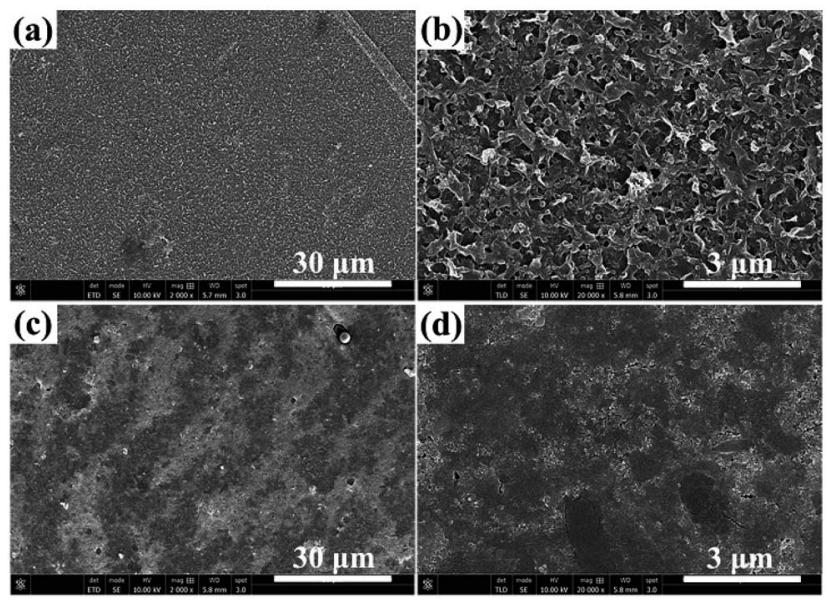

Fig. 11 SEM images of RO membranes after BSA fouling. (a and b) MM6h membrane; (c and d) PA membrane.

the MM-6h membrane, the flux recovery percentage is $97.6 \%$, which is essentially in accordance with flux recovery of MM$\mathrm{COOH}$ membrane.
Table 4 Flux recovery data of RO membranes after fouling and rinsing

\begin{tabular}{lll}
\hline Membrane & $\begin{array}{l}\text { After fouling } \\
(\%)\end{array}$ & $\begin{array}{l}\text { After rinsing } \\
(\%)\end{array}$ \\
\hline PA & 74.6 & 79.2 \\
MM-3h & 80.7 & 89.3 \\
MM-6h & 91.4 & 97.6 \\
MM-12h & 89.1 & 94.8 \\
MM-COOH & 93.7 & 98.5 \\
\hline
\end{tabular}

It has been well demonstrated that surface roughness is related to antifouling ability of membranes. ${ }^{35,50}$ Compared with the mixed-matrix membranes with smooth surface, more declination in flux recovery is observed in the virgin PA membrane with high surface roughness. Generally, foulants are likely to be absorbed in the valleys of membranes with coarser surfaces, which results in clogging of passage for water molecules. ${ }^{51,52}$ Therefore, it is important to fabricate membranes with smooth surfaces for improving fouling resistance. In addition to roughness of the membrane, improved hydrophilicity of mixed-matrix membranes favors antifouling property. The water layer formed on hydrophilic membrane surface can decline hydrophobic-hydrophobic affinity between BSA and membrane surface, which prevents adsorption of proteins on

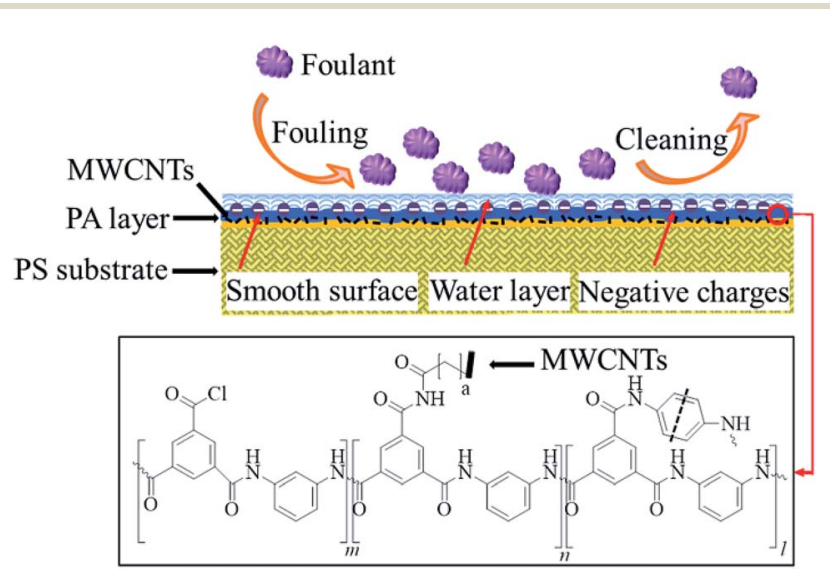

Fig. 12 Antifouling mechanism of mixed-matrix RO membranes. 
membrane surface. ${ }^{53,54}$ Furthermore, it has been generally accepted that membrane surface charge is responsible for protein adsorption on solid surfaces in aqueous solution. ${ }^{27}$ The isoelectric point of BSA is about 4.7, and BSA is negatively charged at neutral $\mathrm{pH}$ in feed solution. ${ }^{55}$ Meanwhile, RO membranes are also negatively charged at $\mathrm{pH}$ 7.0, as shown in Table 1. Thus, a strong electrostatic repulsion force between both negatively charged BSA and membrane surface plays an important role in improving membrane antifouling performance. ${ }^{56}$ The antifouling mechanism of mixed-matrix RO membranes is shown in Fig. 12. As discussed above, it should be noted that PAAm functionalized MWCNTs offer significant changes in membrane antifouling capability due to decrement of surface roughness and enhancement of membrane surface hydrophilicity as well as increment of negative charge.

\section{Conclusions}

In this paper, mixed-matrix membranes with ATRP functionalized MWCNTs were fabricated by interfacial polymerization. DLS and XPS analysis showed that dispersion in aqueous solution and compatibility between MWCNTs and polymeric matrix were improved by grafting hydrophilic and functional PAAm using ATRP method. PAAm functionalized MWCNTs had significant effect on membrane separation and antifouling capability. Notably, MM-6h membrane exhibited a water flux of $48.4 \mathrm{~L} \mathrm{~m}^{-2} \mathrm{~h}^{-1}$, which was $34 \%$ higher than that of virgin PA membrane. More importantly, selectivity performance of MM$6 \mathrm{~h}$ membrane was improved with $\mathrm{NaCl}$ rejection of $98.9 \%$ owing to appropriate grafting time and functional amine groups from PAAm. The problem of decreased salt rejection in RO membrane incorporated with carboxylic MWCNTs was solved. Moreover, mixed-matrix RO membranes showed excellent antifouling performance to BSA. Notably, the MM-6h membrane showed around $97.6 \%$ flux recovery, which is similar to that of MWCNT-COOH membrane with flux recovery of $98.5 \%$. Therefore, this work will be helpful in guiding the design and fabrication of TFC RO membrane with excellent separation and antifouling performance.

\section{Conflicts of interest}

There are no conflicts to declare.

\section{Acknowledgements}

This work was supported by the National Key Research and Development Projects (No. 2017YFC0403903); National Natural Science Foundation of China (No. 21606059) and Special Fund for Basic Scientific Research Business of Central Public Research Institutes (No. K-JBYWF 2016-T10; 2016-T11; 2017T10).

\section{References}

1 M. A. Shannon, P. W. Bohn, M. Elimelech, J. G. Georgiadis, B. J. Marinas and A. M. Mayes, Nature, 2008, 452, 301-310.
2 N. Misdan, W. J. Lau and A. F. Ismail, Desalination, 2012, 287, 228-237.

3 J. R. Werber, C. O. Osuji and M. Elimelech, Nat. Rev. Mater., 2016, 16037.

4 G. D. Kang and Y. M. Cao, Water Res., 2012, 46, 584-600.

5 A. K. Ghosh, B. H. Jeong, X. Huang and E. M. V. Hoek, J. Membr. Sci., 2008, 311, 34-45.

6 J. H. Jhaveri and Z. V. P. Murthy, Desalination, 2016, 379, 137-154.

7 R. Ma, Y. L. Ji, X. D. Weng, Q. F. An and C. J. Gao, Desalination, 2016, 381, 100-110.

8 C. L. Kong, T. Shintani, T. Kamada, V. Freger and T. Tsuru, J. Membr. Sci., 2011, 384, 10-16.

9 S. H. Kim, S. Y. Kwak and T. Suzuki, Environ. Sci. Technol., 2005, 39, 1764-1770.

10 V. Vatanpour and N. Zoqi, Appl. Surf. Sci., 2017, 396, 14781489.

11 Y. Zhang, Y. Wan, G. Pan, H. Shi, H. Yan, J. Xu, M. Guo, Z. Wang and Y. Liu, Appl. Surf. Sci., 2017, 419, 177-187.

12 D. Nikolaeva, C. Langner, A. Ghanem, M. A. Rehim, B. Voit and J. Meier-Haack, J. Membr. Sci., 2015, 476, 264-276.

13 H. J. Kim, Y. Baek, K. Choi, D. G. Kim, H. Kang, Y. S. Choi, J. Yoon and J. C. Lee, RSC Adv., 2014, 4, 32802-32810.

14 Y. Zhang, Z. Wang, W. Lin, H. Sun, L. Wu and S. Chen, J. Membr. Sci., 2013, 446, 164-170.

15 L. Zou, I. Vidalis, D. Steele, A. Michelmore, S. P. Low and J. Q. J. C. Verberk, J. Membr. Sci., 2011, 369, 420-428.

16 J. S. Louie, I. Pinnau, I. Ciobanu, K. P. Ishida, A. Ng and M. Reinhard, J. Membr. Sci., 2006, 280, 762-770.

17 A. Giwa, N. Akther, V. Dufour and S. W. Hasan, RSC Adv., 2016, 6, 8134-8163.

18 J. T. Duan, E. Litwiller and I. Pinnau, J. Membr. Sci., 2015, 473, 157-164.

19 J. Yin, E. S. Kim, J. Yang and B. Deng, J. Membr. Sci., 2012, 423-424, 238-246.

20 S. H. Park, Y. S. Ko, S. J. Park, J. S. Lee, J. Cho, K.-Y. Baek, I. T. Kim, K. Woo and J. H. Lee, J. Membr. Sci., 2016, 499, 80-91.

21 S. Y. Lee, H. J. Kim, R. Patel, S. J. Im, J. H. Kim and B. R. Min, Polym. Adv. Technol., 2007, 18, 562-568.

22 H. S. Lee, S. J. Im, J. H. Kim, H. J. Kim, J. P. Kim and B. R. Min, Desalination, 2008, 219, 48-56.

23 H. Huang, X. Qu, X. Ji, X. Gao, L. Zhang, H. Chen and L. Hou, J. Mater. Chem. A, 2013, 1, 11343-11349.

24 J. Farahbakhsh, M. Delnavaz and V. Vatanpour, Desalination, 2017, 410, 1-9.

25 H. J. Kim, M. Y. Lim, K. H. Jung, D. G. Kim and J. C. Lee, J. Mater. Chem. A, 2015, 3, 6798-6809.

26 H. J. Kim, K. Choi, Y. Baek, D. G. Kim, J. Shim, J. Yoon and J. C. Lee, ACS Appl. Mater. Interfaces, 2014, 6, 2819-2829.

27 H. Zhao, S. Qiu, L. Wu, L. Zhang, H. Chen and C. Gao, J. Membr. Sci., 2014, 450, 249-256.

28 J. Park, W. Choi, S. H. Kim, B. H. Chun, J. Bang and K. B. Lee, Desalin. Water Treat., 2010, 15, 198-204.

29 G. Hummer, J. C. Rasaiah and J. P. Noworyta, Nature, 2001, 414, 188-190.

30 Y. L. Liu, Polym. J., 2016, 48, 351-358. 
31 Y. F. Li, G. W. He, S. F. Wang, S. N. Yu, F. S. Pan, H. Wu and Z. Y. Jiang, J. Mater. Chem. A, 2013, 1, 10058-10077.

32 S. Kar, R. C. Bindal and P. K. Tewari, Nano Today, 2012, 7, 385-389.

33 K. Balasubramanian and M. Burghard, Small, 2005, 1, 180192.

34 A. Hirsch, Angew. Chem., Int. Ed., 2002, 41, 1853-1859.

35 D. Rana and T. Matsuura, Chem. Rev., 2010, 110, 2448-2471.

36 J. H. Choi, J. Jegal and W. N. Kim, J. Membr. Sci., 2006, 284, 406-415.

37 M. Ramanujam, R. Mix, M. Wagner and J. Friedrich, J. Adhes. Sci. Technol., 2013, 27, 1828-1839.

38 J. Jiang, Y. Zhang, X. Guo and H. Zhang, Macromolecules, 2011, 44, 5893-5904.

39 Y. Liu, C. Y. Nie, X. J. Liu, X. T. Xu, Z. Sun and L. K. Pan, RSC Adv., 2015, 5, 15205-15225.

40 B. W. Zhang, L. X. Liu, S. Y. Xie, F. Shen, H. Yan, H. H. Wu, Y. H. Wan, M. Yu, H. J. Ma, L. F. Li and J. Y. Li, RSC Adv., 2014, 4, 16561-16566.

41 T. Wang, L. Dai, Q. Zhang, A. Li and S. Zhang, J. Membr. Sci., 2013, 440, 48-57.

42 M. Ghanbari, D. Emadzadeh, W. J. Lau, T. Matsuura and A. F. Ismail, $R S C A d v .$, 2015, 5, 21268-21276.

43 E. Igbinigun, Y. Fennell, R. Malaisamy, K. L. Jones and V. Morris, J. Membr. Sci., 2016, 514, 518-526.

44 H. Z. Shafi, Z. Khan, R. Yang and K. K. Gleason, Desalination, 2015, 362, 93-103.
45 Ihsanullah, A. M. Al Amer, T. Laoui, A. Abbas, N. Al-Aqeeli, F. Patel, M. Khraisheh, M. A. Atieh and N. Hilal, Mater. Des., 2016, 89, 549-558.

46 P. Daraei, S. S. Madaeni, N. Ghaemi, M. A. Khadivi, B. Astinchap and R. Moradian, J. Membr. Sci., 2013, 444, 184-191.

47 P. G. Rouxhet, A. M. Misselyn-Bauduin, F. Ahimou, M. J. Genet, Y. Adriaensen, T. Desille, P. Bodson and C. Deroanne, Surf. Interface Anal., 2008, 40, 718-724.

48 J. M. Martin, C. Grossiord, T. Le Mogne and J. Igarashi, Tribol. Int., 2000, 33, 453-459.

49 W. F. Chan, E. Marand and S. M. Martin, J. Membr. Sci., 2016, 509, 125-137.

50 J. L. Wang, X. L. Gao, J. Wang, Y. Wei, Z. K. Li and C. J. Gao, ACS Appl. Mater. Interfaces, 2015, 7, 4381-4389.

51 A. Razmjou, J. Mansouri and V. Chen, J. Membr. Sci., 2011, 378, 73-84.

52 E. M. Vrijenhoek, S. Hong and M. Elimelech, J. Membr. Sci., 2001, 188, 115-128.

53 L. Zhao, P. C. Y. Chang, C. Yen and W. S. W. Ho, J. Membr. Sci., 2013, 425-426, 1-10.

54 J. Wang, X. L. Gao, Q. Wang, H. J. Sun, X. J. Wang and C. J. Gao, Appl. Surf. Sci., 2015, 356, 467-474.

55 L. Liu, D. Y. W. Di, H. Park, M. Son, H. G. Hur and H. Choi, RSC Adv., 2015, 5, 7340-7348.

56 I. H. Huisman, P. Prádanos and A. Hernández, J. Membr. Sci., 2000, 179, 79-90. 